\title{
Mining association rules from structured XML data
}

\begin{abstract}
XML has become the standard for data representation on the web. This expansion in reputation has prompted the need for a technique to access XML documents. Many techniques have been proposed to tackle the problem of mining XML data. We study the various techniques to mine XML data and yet We presented a java based implementation of FLEX algorithm for mining XML data.
\end{abstract}

Keyword: Data mining; Association rules; XML; Java 